\title{
New Directional Morphological Approaches for the Characterization of Fingerprints
}

\section{Nuevos métodos morfológicos dereccionales para la caracterización de huellas digitales}

\author{
L.A. Morales-Hernández \\ Universidad Autónoma de Querétaro, Facultad de Ingeniería. \\ San Juan del Río, Querétaro. \\ E-mail:luis_morah@yahoo.com \\ I.R. Terol-Villalobos \\ CIDETEQ, Parque Tecnológico Querétaro, Querétaro. \\ E-mail:famter@ciateq.net.mx \\ A. Domínguez-González \\ Universidad Autónoma de Querétaro, Facultad de Ingeniería. \\ San Juan del Río, Querétaro. \\ E-mail:auredgz@uaq.mx \\ G. Herrera-Ruiz \\ Universidad Autónoma de Querétaro, Facultad de Ingeniería. \\ San Juan del Río, Querétaro. \\ E-mail:gherrera@uaq.mx
}

(Recibido: octubre de 2007; aceptado: agosto de 2008)

\begin{abstract}
The present paper proposes two methods to characterize fingerprints. The first method estimates the main orientations of the structures of ridges and valleys. This proposed approach detects the preferential directions that characterize the fingerprint as orientation fields using directional granulometries. This approach allows to have a better characterization by means of two parameters; the sizes and the orientations of the structures contained in the fingerprint. In order to achieve the characterization of the fingerprint orientations, the directional morphology is applied to detect the regions of same orientation. Then, the morphological transformations with line segments as structuring elements are applied using a quadtree structure to go from global approach to a local one. The second method uses directional erosions to build two images that contain the sizes and orientations of the fingerprint. Then the rank-max connected opening is applied to filter the image containing the sizes of the ridges in order to select the main structures. Here it is illustrated that the obtained information enables us to reconstruct the directional behavior of the fingerprint. The proposed methods are applied to characterize the four typical classes of fingerprintswith excellent results.
\end{abstract}

Keywords: Characterization, fingerprints, directional morphological, directional granulometries, rank-maxconnectedopenning, quadtree.

\section{Resumen}

El presente artículo propone dos nuevos métodos para la caracterización de huellas digitales. El primero estima las principales orientaciones de las estructuras de crestas y valles. 
DOI: http://dx.doi.org/10.22201/fi.25940732e.2009.10n3.024

New Directional Morphological Approaches for the Characterization of Fingerprints

Este método propuesto detecta las direcciones preferenciales en la huella digital, caracterizando a éstas como campos direccionales mediante granulometrías direccionales. Esta metodologia permite tener una mejor caracterización mediante dos parámetros de las estructuras contenidas en la huella digital, como son el tamaño de las crestas y sus respectivas orientaciones. Para lograr la caracterización de las orientaciones de la huella digital se aplican técnicas de morfología direccional, buscando zonas con la misma orientación. Para la caracterización de las crestas se aplica la morfología matemática mediante segmentos de recta como elementos estructurales usando la metodología de la estructura quadtree para ir de una aproximación global a una local. El segundo método emplea erosiones direccionales para construir dos imágenes que contienen los tamaños y las orientaciones principales. Después es aplicada la apertura conexa de rango máximo para filtrar la imagen que contiene los tamaños de las crestas con el fin de seleccionar las estructuras principales. Aqui es ilustrado que a partir de esta información es posible reconstruir el comportamiento de las estructuras direccionales de la huella. Los métodos propuestos son aplicados a las clases típicas de huellas digitales mostrando excelente resultados en la caracterización.

Desciptores: Caracterización, huellas digitales, morfología direccional, granulometrías direccionales, apertura conexa de rango máximos y cuadrantes.

\section{Introduction}

Fingerprints are today the most widely used in biometric features for personal identification. With increasing emphasis on identity management, automatic fingerprint recognition has commercially received wide attention. Nevertheless, there are still some critical research issues such as fingerprint classification and identification. Both topics play an important role in orientation patterns, which can be defined by a local direction of ridge-valley structures, (Jain et al., 1997a), (Maoi and Maltoni, 1997), (Cappelli et al., 1999), (Jiang and Yau, 2000), (Greenberg and Kogan, 2006).

A typical fingerprint recognition system consists of three main components: (1) orientation field estimation; (2) ridge enhancement and tinning; (3) minutiae extraction (Jain et al., 1997b). Several methods have been proposed to improve the estimation of the orientation field, which can broadly be categorized as filtering-based (Jain et al., 1997b), (Maoi and Maltoni, 1997), (Khan et al., 2005) and model-based (Sherlock and Monro, 1993), (Vizcaya and Gerhardt, 1996). Most of these methods rely on a number of fixed possible templates or filters (Maltoni et al., 2003). Recently, some works for fingerprint orientation field, based on constrained nonlinear models that require optimal estimators has been proposed (Sherlock and Monro, 1993), (Vizcaya and Gerhardt, 1996), (Zhou and Gu, 2004), (Li et al., 2006). However, these methods cannot be applied to accurately approximate real fingerprint orientation field because do not consider the influence of a singular point.

The main disadvantage of mathematics methods, for instance the Bayesian networks (Dass, 2004), complex polynomials (Zhou and $\mathrm{Gu}, 2004$ ) and rational polynomials (Sherlock and Monro, 1993), is that they need to know the locations and types of singularities in ridge pattern in order to adjust the system parameters. Singular points are the points in a fingerprint where the directional field is discontinuous. An example of their application is the classification of fingerprints in the Henry classes (1900). The orientation of singular points can be also used to initialize flow lines in the directional field (Kawagoe and Tojo, 1984), (Karu and Jain, 1996), (Chong et al., 1997), (Bazen and Gerez, 2002).

Other researches use the orientation field estimation for different applications. Olivera and Laite (2007) propose extracts the orientation field of an image for reconnecting broken ridges in fingerprints images. Kulkarni et al. (2006) apply the orientation field for fingerprint verification using image-based technique in which the variance feature of orientation field is used for matching. The test and trainee fingerprint images are aligned using the obtained orientations using an invariant rotation algorithm. To do it, the first stage is to find the core point. Qi and Wang (2005) combine the global orientation field with minutia where each fingerprint minutia is related with the orientation field. This method use the orientation field estimation algorithm proposed by Jain et al. (1997b). 
The present paper is focused on the application of mathematical morphology methodology to model orientation fields. Particularly, the study will focus on the characterization of the fingerprints by using directional morphological transformations. Since fingerprints can be considered as a structure composed by a set of line segments, a bank of filters which is composed by directional morphological transformations permit to extract the main orientations of the image. This is similar to the perception of the orientation of line segments by the human brain. In order to achieve an image processing oriented to these image structures, two approaches are investigated in this paper. The first one is a global approach based on the directional granulometries which is computed by morphological openings using directional structural elements also called line segments. This approach allows determining the main directions of the structures by studying the minima of the granulometric density function. In order to define a local approach, a quadtree structure is used to decompose the image with a different resolution that is according to the levels of the tree. Thus, it is possible to obtain a multi-scale local approach to define descriptors that considers the size or scale and orientation.

The second method considers a local approach by using the concept of distance function which is computed by the supremum of directional erosions. Thus, the maxima of the distance function contain the information of the biggest structural elements or line segment that can be placed inside the structure. In order to know the orientations, a second image is computed by detecting the orientation of the supremum of directional erosions. These local descriptors for the size and orientation provide an excellent reconstruction of the orientations and allow describing the model of the fingerprint orientation pattern in a piecewise manner. The main advantage of this is algorithm is that it is able to predict orientation in a more efficiently way because the proposed approach does not need to determine the core or delta of the fingerprint.

\section{Some basic concepts of mathematical morphology}

Morphological filters are increasing and idempotent transformations (Serra, 1988), (Heijmans, 1994), (Soi1le, 2003). While the increasing property expresses that the order is preserved, a transformation $\Psi$ is idempotent if and only if for all functions $f, \psi(\psi(f))=\psi(f)$. The basic morphological filters are the morphological opening $\gamma_{\mu B}$ and the morphological closing $\varphi_{\mu B}$ with a given structuring element $\mu B . B$ is the transposed set $(B=\{-x: x \in B\}$ and $\mu$ is an homothetic (scale) parameter. In this work, the homothetic parameter only takes integer values. The morphological opening is an anti-extensive filter and the morphological closing is an extensive filter. These transformations are expressed by the morphological dilation $\delta_{\mu B}$ and morphological ero$\operatorname{sion} \varepsilon_{\mu B}$. Thus,

$$
\begin{aligned}
& \gamma_{\mu B}(f)(x)=\delta_{\mu B}\left(\varepsilon_{\mu B}(f)\right) \\
& \varphi_{\mu B}(f)(x)=\varepsilon_{\mu B}\left(\delta_{\mu B}(f)\right) .
\end{aligned}
$$

The morphological erosion and dilatation are respectively expressed by: $\varepsilon_{\mu B}(f(x))=\wedge\left\{f(y) ; y \in \mu B_{x}\right\}$ and $\delta_{\mu B}(f(x))=\vee\left\{f(y) ; y \in \mu B_{x}\right\}$, where $\wedge$ is the infimum operator and $\vee$ is the supremum operator. Another interesting class of filters is composed by the openings and closings by reconstruction. When filters by reconstruction are built, the geodesic dilation and geodesic erosion of size 1 are iterated until idempotence is reached (Soille, 2003). Where the geodesic dilation and the geodesic erosion of size one are given by $\delta_{f}^{1}(g)=f \wedge \delta_{B}(g)$ with $f \geq g$ and $\varepsilon_{f}^{1}(g)=f \vee \varepsilon_{B}(g)$ with $f \leq g$, respectively. When the function $g$ (the marker) is equal to the dilation or to the erosion of the original function by a given structuring element, the closing and the opening by reconstruction are obtained.

$$
\begin{aligned}
& \bar{\varphi}_{\lambda B}(f)=\lim _{n \rightarrow \infty} \varepsilon_{f}^{n}\left(\delta_{\lambda B}(f)\right)=\varepsilon_{f}^{1} \varepsilon_{f}^{1} \ldots \varepsilon_{f}^{1}\left(\delta_{\lambda B}(f)\right) \\
& \bar{\gamma}_{\lambda B}(f)=\lim _{n \rightarrow \infty} \delta_{f}^{n}\left(\varepsilon_{\lambda B}(f)\right)=\delta_{f}^{1} \delta_{f}^{1} \ldots \delta_{f}^{1}\left(\varepsilon_{\lambda B}(f)\right) .
\end{aligned}
$$

\section{Directional supremum of directional erosions and directional granulometry}

In mathematical morphology there are some operators based on the detection of the residues of parametric transformations; for example, the ultimate erosion, the morphological skeleton, the granulometry function and the distance function.

These two last transformations are computed by the difference of successive openings or erosions, respectively. Generally, an associated function is linked to these transformations; for example the density or the distribution functions are related to the granulometry residues. Granulometry (anti-granulometry) was formalized by Matheron (1975), for binary images and extended to complete lattices by Serra (1988). Now, the directional granulometry and a function associated to the directional erosions are presented 
DOI: http://dx.doi.org/10.22201/fi.25940732e.2009.10n3.024

New Directional Morphological Approaches for the Characterization of Fingerprints

\section{Definition 1}

A family of openings $\left\{\gamma_{\lambda}\right\}$ (or respectively of closings $\left\{\varphi_{\lambda}\right\}$ ) where $\lambda \in\{1, \ldots n\}$, is a granulometry (respectively anti-granulometry) if for all $\lambda, \mu \in\{1, \ldots, n\}$ and all func$\operatorname{tion} f, \lambda \leq \mu \Rightarrow \gamma_{\lambda}(f) \geq \gamma_{\mu}(f)$ (respectively $\varphi_{\lambda}(f) \leq \varphi_{\mu}(f)$ ).

The above definition implies that for greater parameter $\mu$; the opening (closing) becomes more severe. The granulometric analysis of a binary or gray-level image, consists of associate each $\mu_{i}$ value a measure of the image $\gamma_{\mu i}(f)$. When a granulometry study is made based on the openings, two functions are associated to these transformations; the granulometric density function $g$ and its distribution function $G$ given respectively by:

$$
\begin{aligned}
& g_{\lambda}(f)=\left(\operatorname{Mes}\left(\gamma_{\lambda}(f)\right)-\operatorname{Mes}\left(\gamma_{\lambda+1}(f)\right)\right) / \operatorname{Mes}(f) \\
& G_{\lambda}(f)=\left(\operatorname{Mes}(f)-\operatorname{Mes}\left(\gamma_{\lambda}(f)\right)\right) / \operatorname{Mes}(f),
\end{aligned}
$$

where Mes represents the volume for gray-level images and the area for binary images.

Since the interest in this paper is to introduce of directional granulometry and a function computed by the supremum of directional erosions in order to characterize the fingerprints, the size of the structural element $\lambda$ and the angle $\alpha$ (direction) parameters are required to characterize them. Thus, the set of points of a line segment is computed by:

If $\alpha \leq 45^{\circ}$ then, $y_{i}=x_{i} \tan \alpha$ for $x_{i}=0,1, \ldots, \lambda \cos \alpha$

If $\alpha>45^{\circ}$ then $x_{i}=y_{i} \cot \alpha$ for $y_{i}=0,1, \ldots, \lambda \sin \alpha$.

Then, morphological opening and closing filters are given by:

$$
\gamma_{L_{\alpha, \lambda}}(f)=\delta_{L_{\alpha, \lambda}} \varepsilon_{L_{\alpha, \lambda}}(f), \quad \varphi_{L_{\alpha, \lambda}}(f)=\varepsilon_{L_{\alpha, \lambda}} \delta_{L_{\alpha, \lambda}}(f),
$$

where the morphological erosion and dilation filters are given by:

$$
\begin{aligned}
& \varepsilon_{L_{\alpha, \lambda}}(f)(x)=\min \left\{f(y): y \in L_{\alpha, \lambda}(x)\right\} \\
& \delta_{L_{\alpha, \lambda}}(f)(x)=\max \left\{f(y): y \in L_{\alpha, \lambda}(x)\right\} .
\end{aligned}
$$

When a directional erosion size $\lambda$ is applied to an image at all directions, some output computed images for some directions contain more information of the original image depending of the anisotropies presented in the image. Since the objective of applying directional erosions consists in preserving the maximum of information for all directions, the supremum of the erosions is computed by the following relationship.

$$
\varepsilon_{\lambda}^{\text {sup }}(X)={ }^{\cup \varepsilon} L_{\substack{\alpha, \lambda \\ \alpha \in[0,180]}}(X) .
$$

Whereas computing the directional density function $g(\alpha, \lambda)$ one obtains the portion of the structure, for a given direction $\alpha$, of size $\lambda$, the directional distribution function $G(\alpha, \lambda)$ gives the fraction of the structures greater or equal to the length $\lambda$ in the direction $\alpha$.

$$
\begin{aligned}
& g(\alpha, \lambda)(f)=\left(\operatorname{Mes}\left(\gamma_{L_{(\alpha, \lambda)}}(f)\right)-\operatorname{Mes}\left(\gamma_{L_{\alpha, \lambda+1}}(f)\right)\right) / \operatorname{Mes}(f) \\
& G(\alpha, \lambda)(f)=\left(\operatorname{Mes}(f)-\operatorname{Mes}\left(\gamma_{L_{(\alpha, \lambda)}}(f)\right)\right) / \operatorname{Mes}(f) .
\end{aligned}
$$

For the sake of simplicity the structuring element is expressed by $\alpha, \lambda$ in $g$ and $G$.

The importance of this function is that it permits the detection of the main structures in a given direction. Thus, instead of fixing the parameter $\alpha$, the parameter $\lambda$ was fixed (directional granulometry).

\section{Application of fingerprint orientation field based on directional granulometry and quadtree structure}

In this section, the application of quadtree structure is presented for left loop, right loop, worth loop and arch loop classes of fingerprints (Henry, 1900). The left loop fingerprint class is presented in detailed way and for the other cases it is presented only the final results.

In mathematical morphology, an important work for directional filtering was proposed in (Soille et al., 2001). In the present paper a morphological approach that uses the concept of directional granulometries and the quadtree structure is proposed in order to characterize anisotropies in the images.

The figure $1 \mathrm{~b}$ illustrates the distribution function of the image in figure $1 \mathrm{a}$ for $\lambda=80$ and $0 \leq \alpha<180$. The minimum of this function permits to determine the direction of the main structures. The minimum in figure $1 \mathrm{c}$ was computed from the function in figure $1 \mathrm{~b}$ using morphological transformations in one-dimensional case. To carry out the minimum detection, the distribution function was transformed into the interval $[0,255]$ in integer numbers and then, the typical morphological tools for detecting minima in mathematical morphology was 
applied (Soille, 2003). In fact, the minima of the image will enable to have a criterion to go from a global approach to a local one by the quadtree structure. In the quadtree approach, the coding by regions is made by a homogeneity criterion that enables to discriminate whether a squared region can be considered as a connected component. The process starts with a square of $2^{\mathrm{n}}$ pixels that is divided in four squared zones. Each squared zone is studied on the original image using one or several homogeneity criteria (variance, max-min values, etc). If the homogeneity criterion is verified, a function value is given at all points of the square region; for instance, the average of the intensity values in the square. For any square that does not verify the homogeneity criterion, a similar procedure is performed in a recursive way by dividing the squared region by four. For orientation fields, it is clear that a homogeneity criterion is given by only one direction. In our case, the minima of the distribution function are employed as criterion. If the distribution function in a squared region presents only a principal minimum, then the region is considered homogeneous. In this case, the pixel values of the region are affected by the angle of the minimum where the minimum was found. Otherwise, if the distribution function of a square region has several representative minima, then the region is divided by four. The figures 2 and 3 illustrate the approach to determine the orientation field in the image. After dividing the image by four, their distribution functions were computed as illustrated in figure 2. In particular, it is observed that the distribution functions in figures 2, that correspond to the bottom left (O3) and right (Q4) squares, both contain only one principal minimum, while the other two squares contain several representative minima(O1 and Q2). Thus, these last two squares were divided by four and their distribution functions were computed to know their directional homogeneity. The image in figure 3 shows the orientation fields (gray level).

Figure $3 \mathrm{a}$ shows the orientations of the first hierarchy of the quadtree (four square zones), and figures $3 \mathrm{~b}$ and $3 \mathrm{c}$ illustrate the up squared regions divided by four squared regions. Finally, figure $3 \mathrm{~d}$ illustrates the final hierarchy. In figure $3 e$, the regions were affected by a color in order to better illustrate the orientation fields. The color representation is the classical hue definition of the perceptual color spaces.

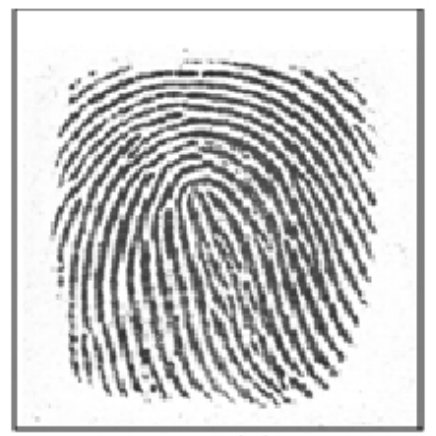

(a)

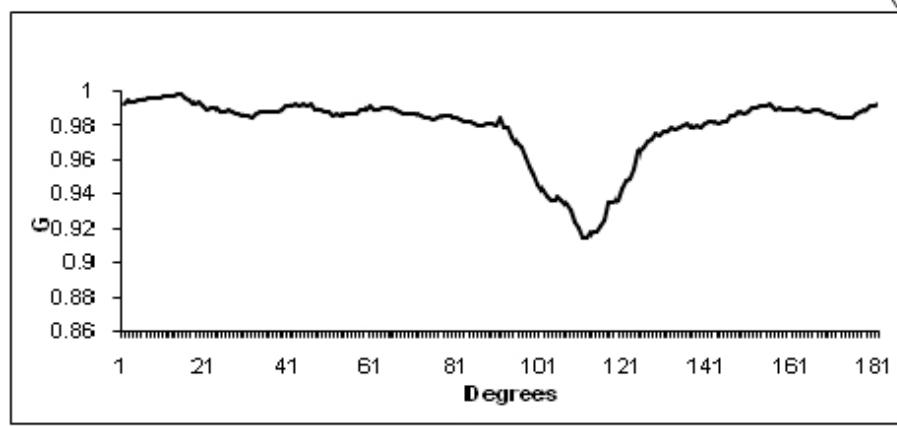

(b)

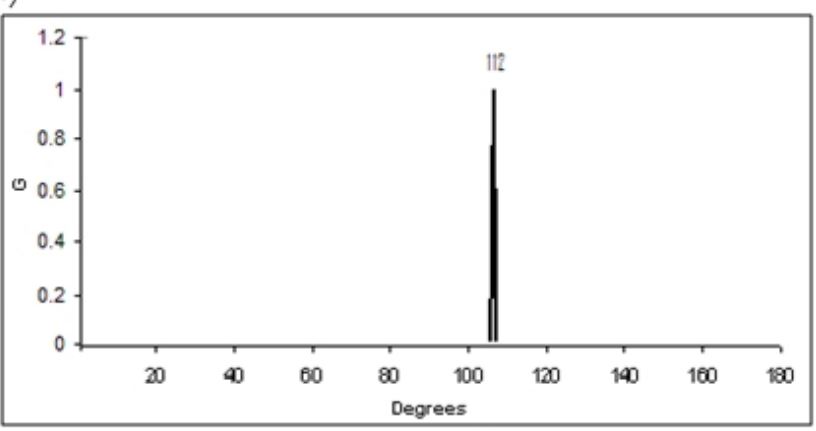

(c)

Figure 1. a) Original image of left loop fingerprint class, b) Directional granulometry size 60, c) Minimum detection (112 degrees) 


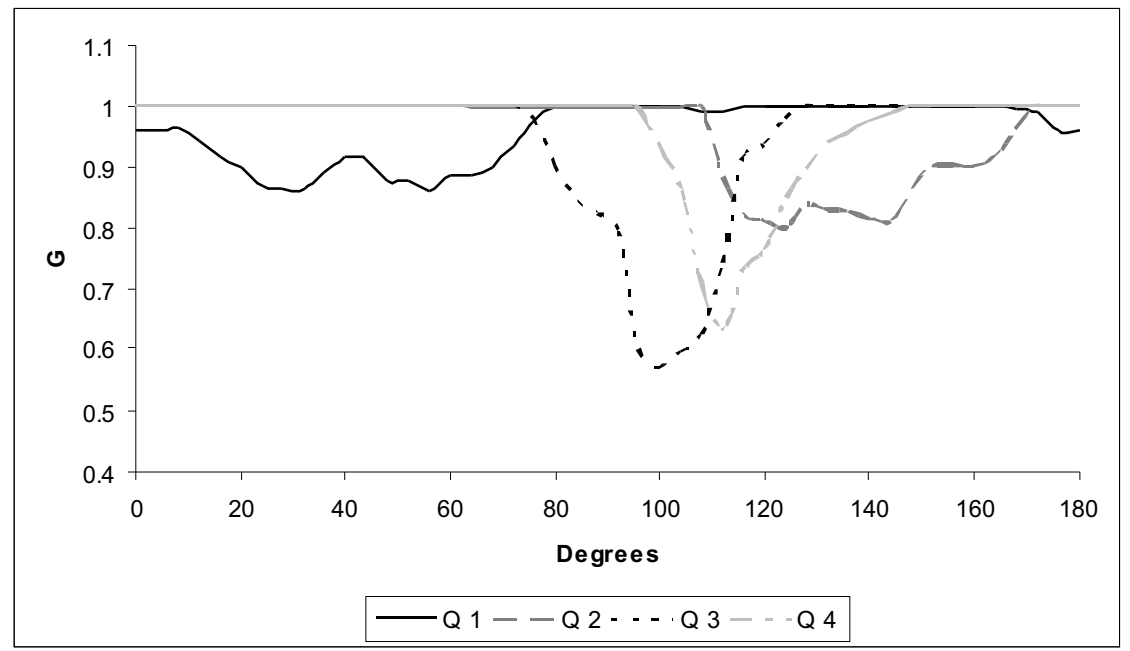

Figure 2. Directional granulometries at first level of the hierarchy: Q1 and Q2 Top squares regions, Q3 and Q4 bottom square regions

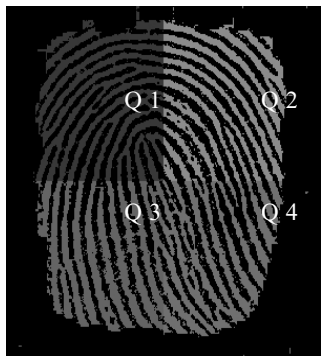

(a)

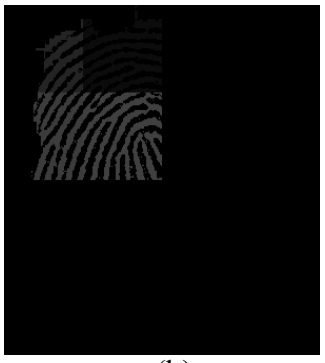

(b)

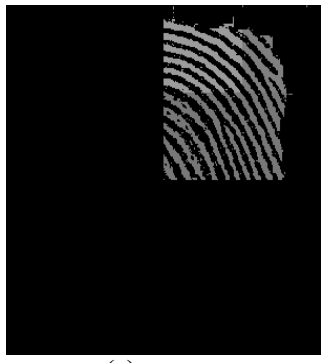

(c)

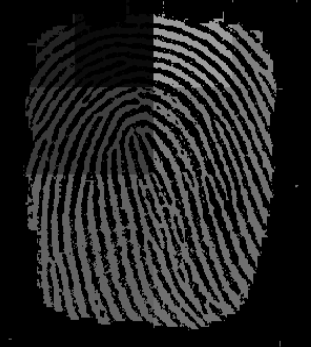

(d)

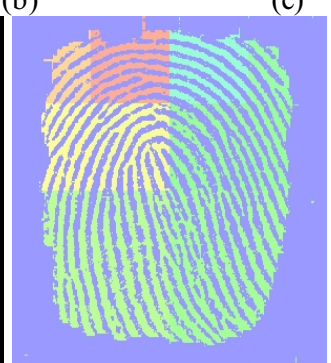

(e)

Figure 3. a) First hierarchy of the quadtree, b) and c) Second hierarchy of the quadtree, d) Final quadtree structure, e) Color representation of the quadtree

In summary, the directional granulometry, also called pattern spectrum (Maragos, 1989), allows to know the size and the predominant orientation in the quadtree at a given scale. The process of the image division in square blocks is finished when only one minimum is found. The image is divided in subimages in order to find the predominant orientation. Then, we can reconstruct the fingerprint image according to the quadtree since each one has the size information and orientation.

Now the application of the quadtree is presented briefly for right loop, worth loop and arch loop classes fingerprints. By assuming a size of 60 on the granulometric distribution function illustrated in Figure 4a; the minima of this function enable us to take the decision to stop the split procedure of the quadtree approach or 
to continue the procedure by splitting the square region. The curve in figure $4 \mathrm{a}$ computed from the square region $\mathrm{Q} 2$ has the most notable minimum, that in fact characterize the main directional structure (the biggest one) contained in the image. In other words, the principal directional structure in the fingerprint is computed at the first hierarchy of the quadtree Q2. In this example, the structural information given by this region of the fingerprint enables us to classify it as a left loop. Figure $4 \mathrm{~d}$ shows the output image containing the final result of the segmenting process. Observe that the granulometric function illustrated in figure $4 a$ of the squared region $\mathrm{Q} 4$, that contains small variations with several minima is correctly segmented. In this case a second hierarchy level is required to achieve the segmentation of the region. Thus, the global behavior at the first hierarchy of the quadtree structure permits to extract the characteristics to classify the fingerprints. This coarser to finer approach inherent in the quadtree permits to better understand the directional structures at each level of the hierarchy based on the granulometric functions.

In the following, the application of the approach based on the quadtree and the directional granulometry for segmenting images, is shown. Three types of fingerprints are segmented by our method; a right loop, a left loop and a whorl. The right and left loops examples in figure $5 \mathrm{a}$ and $5 \mathrm{~d}$ present a similar behavior as that illustrated before in figure $4 \mathrm{a}$. Both fingerprints contain a main directional structure that is detected at the first hierarchy levels of the quadtree (the granulometric distribution functions present a main minimum). This means, these fingerprints can be classified at coarser hierarchies of the quadtree. In the case of the class whorl illustrated in figure $5 \mathrm{~g}$, the fingerprint does not contain a main structure, represented in the granulometric distribution function by a principal minimum. Thus, it can not be classified at coarser scales, and in this case, the finer levels of the hierarchy are required to classify it. These examples show that the hierarchical evolution of the quadtree is linked to the structural characteristics of the fingerprints.

\section{Application of finger print orientation field based on the supremum of directional erosions}

In this section a second approach based on directional erosions to characterize fingerprints is introduced. The idea of applying directional erosions consists in preserving the maximum of information for all directions. To stock the information for all $\lambda$ value, a gray level image

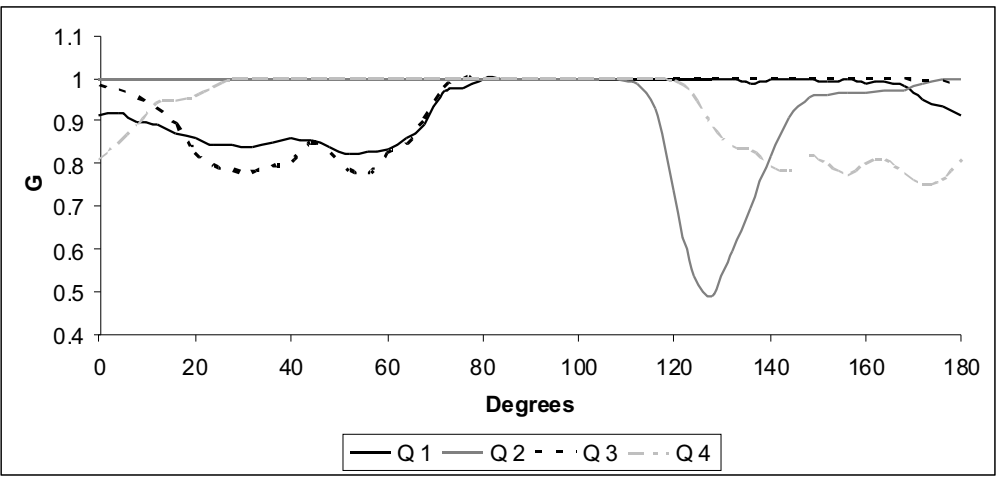

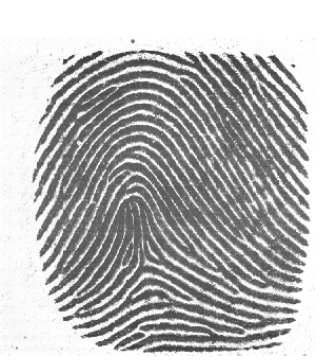

(b)

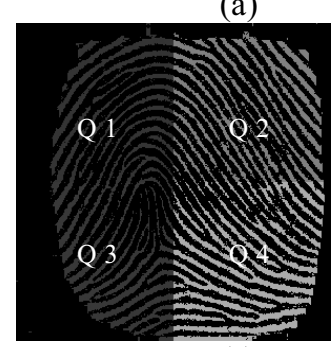

(c)

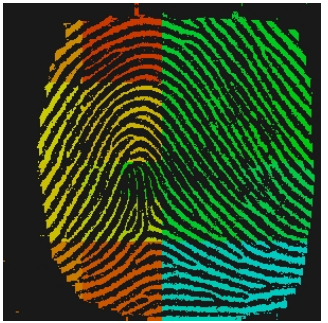

(d)

Figure 4. a) Directional granulometry first hierarchy of the quadtree, b) Original image,

c) First hierarchy of the quadtree, d) Final quadtree color representation 
is used in a similar way that the distance function is built. This means that the procedure is begun for $\lambda=1$ by increasing by one all point $x$ belonging to $\varepsilon_{\lambda}^{\text {sup }}(x)$. Then, the procedure continue until one has a $\lambda_{\max }$ value such that $\varepsilon_{\lambda}^{\text {sup }}(x)=\varnothing$. The maxima of this function are the loci of maximal structuring elements (ultimate erosions). Thus, the positions of the greatest structuring elements that can be completely included in the structure are known but the angles of these structuring elements are unknown. To overcome this problem, a second image containing the angle of the structuring elements is built. The scheme in figure 6 illustrates the procedure for extracting the directional information of the image. First, the tophat size 4 was computed on the original image (figure 1a) for obtaining a binary one figure $7 \mathrm{a}$ (pre-processing). Then, at each size of the structuring element, the supremum of directional erosions for all orientations is computed in order to obtain the distance function and the orientation function illustrated in figures $7 b$ and $7 c$ respectively. Thus, these functions are employed to compute the segment of lines that characterize the structures. The maxima of distance function is computed by obtaining the loci of maximal structuring elements (ultimate erosions) and the values of the orientation function enable to know the orientation lines.

Since, the number of maxima is too much, a filtering process using a class of rank-max connected filters was carried out on the distance function. For obtaining the line segments representation as illustrated in figures $7 \mathrm{~d}$ and $7 \mathrm{e}$. This filtering process will be described below.

\section{Rank-max connected opening}

The morphological opening presents some problems when images have thin regions. Figure $8 b$ illustrates an example of the morphological opening (size 1) applied to an image containing thin regions as that illustrated in figure 8a. A solution of this inconvenience consists it changing the morphological opening. We know that a class of non-linear filters, called rank filters, exhibit

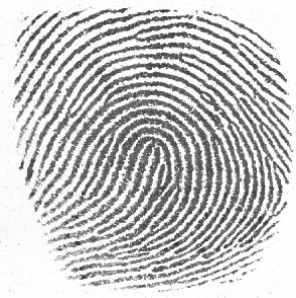

(a)

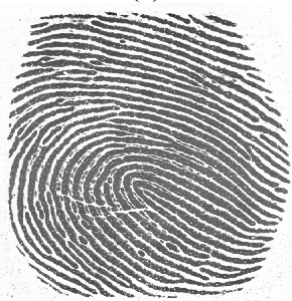

(d)

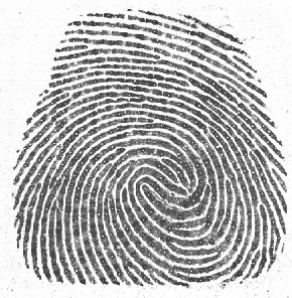

(g)

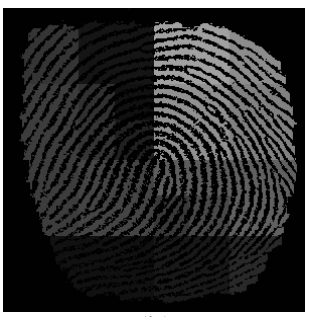

(b)

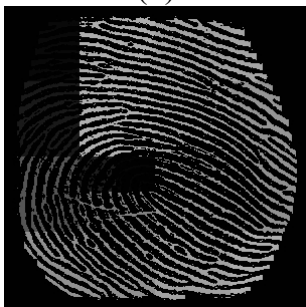

(e)

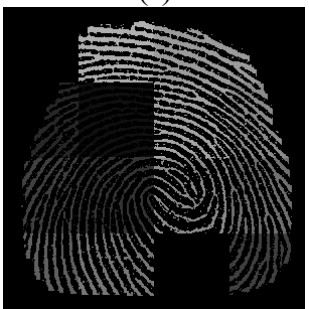

(h)

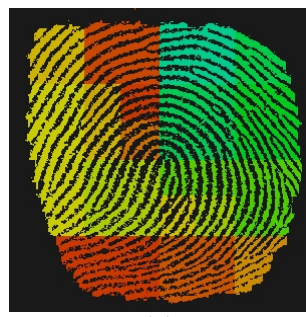

(c)

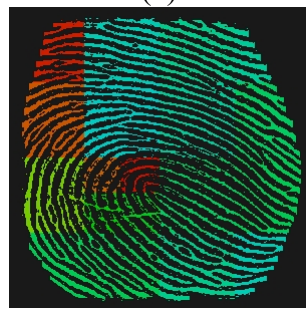

(f)

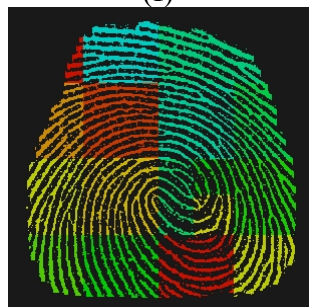

(i)

Figure 5. a) Fingerprint type right loop, b) Final hierarchy of the quadtree, c) Final Quadtree color representation, d) Fingerprint type left loop, e) Final hierarchy of the quadtree, f) Final Quadtree color representation, g) Fingerprint type whorl, h) Final hierarchy of the quadtree, i) Final Quadtree color representation 
excellent robustness properties and provide solutions in many cases where other transformations are inappropriate. Moreover, rank filters are used to build an interesting class of transformations called rank-max openings. Ronse (Serra, 1988) introduced the rank-max openings on sets and gray-level functions as a generalization of the morphological opening by an structuring element $\lambda B$. As follow $\boldsymbol{B}_{k}$ is the set of all subsets $B_{i} \subseteq \lambda B$ containing $k$ points. In the binary case, the rank-max opening is given by,

$$
\gamma \lambda_{I B, k}(X)=\bigcup_{i}\left\{\gamma \psi_{\lambda B_{i}}(X): \lambda B_{i} \subset \lambda B,\left|\lambda B_{i}\right|=k\right\} .
$$

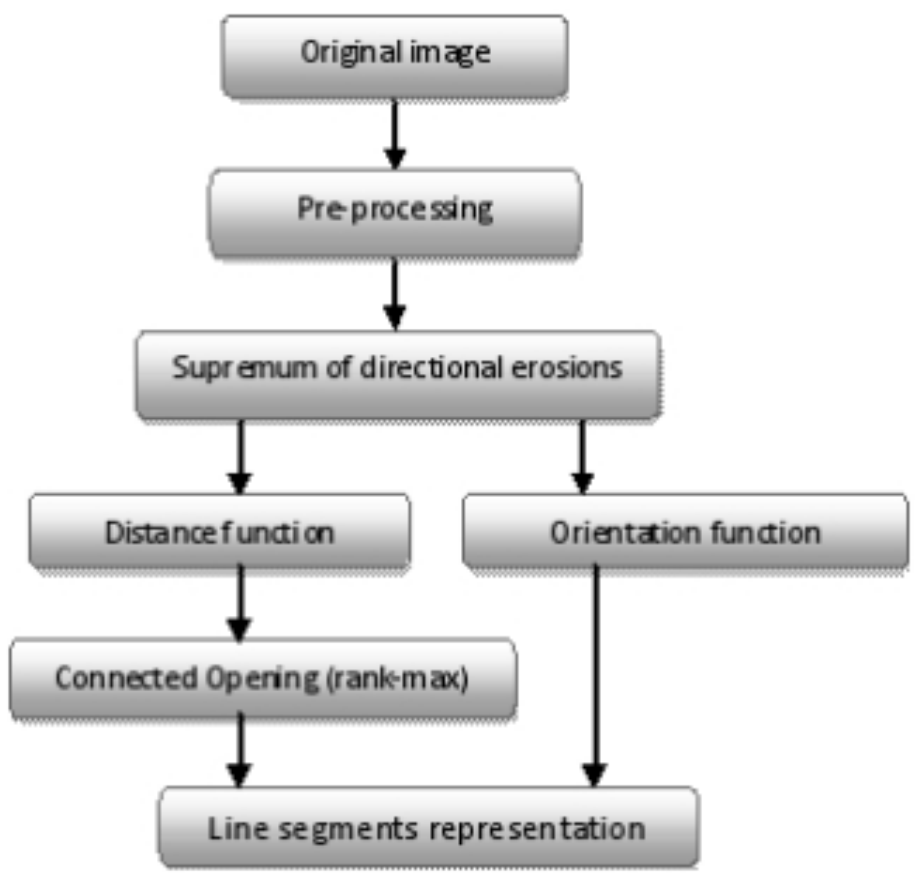

Figure 6. Procedure scheme for computing the orientation field

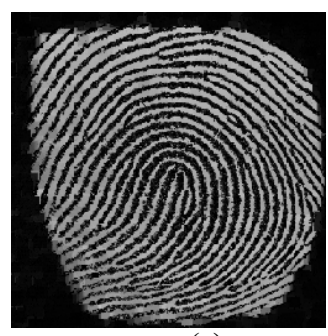

(a)

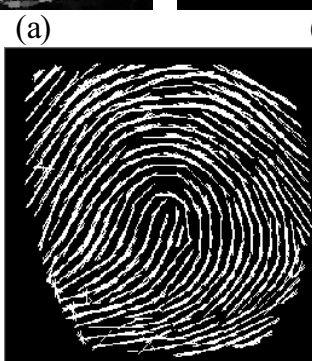

(d)

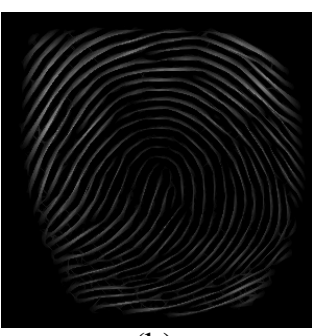

(b)

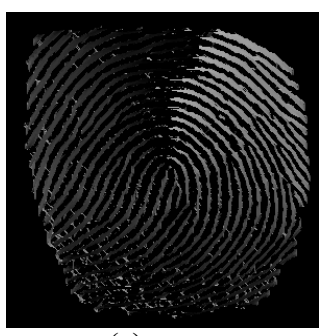

(c)

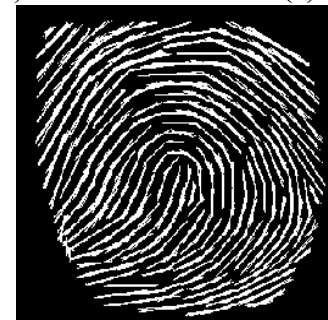

(e)

Figure 7. a) Tophat 4, b) Image distance funtion, c) Image orientation function, d) Image dilation anisotropy connectec 4, e) Image dilation anisotropy connectec 6 
The output image is composed by the union of the images computed by the openings using structuring elements $\lambda B_{i} \subset \lambda B$, where the number of points of the structuring elements is $k$ (cardinality $k,\left|\lambda B_{i}\right|=k$ ). The set of the structuring elements of size $k$ is given by $B_{k}=\left\{\lambda B_{i}: \lambda B_{i} \subset \lambda B\right.$ and $\left.\left|\lambda B_{i}\right|=k\right\}$. Figure 9a illustrates a structuring element $\lambda B_{i}$ with $\lambda=3$ and $\left|\lambda B_{i}\right|=49\left(\lambda B_{i}=\lambda B\right)$, whereas figures $9 \mathrm{~b}-9 \mathrm{~g}$ show six configurations of $\lambda B_{i}$ with $\lambda=3$ and $\left|\lambda B_{i}\right|=16$. The origin of the structuring elements is presented light gray color.

The white color pixels do not belong to the structuring element. Observe that the configurations in figures $9 b-9 d$ contain more than 1 connected component, while the configurations in figures $9 \mathrm{e}-9 \mathrm{~g}$ are composed of only one connected component.

The rank-max opening is more flexible than the morphological opening because it preserves the regions which contain at least $k$ points. This opening transforms a binary image $X$ into the supremum of all portions of it, consisting of "sufficiently large" subset of a translate of $\lambda B$. However, the main drawback of the rank-max opening lies in the interactions between the connected components. This problem leads to a degradation of the filtered image. Figure $8 c$ shows a case when the rank-max opening.

A solution to this problem can be achieved by introducing the connectivity notion. Consider a subset $\boldsymbol{B}_{C k}$ of $\boldsymbol{B}_{k}$ containing all connected subsets of $\boldsymbol{B}_{k}$ which include the origin of $\lambda B\left(B C_{k}=\left\{\lambda B_{i}: \lambda B_{i} \in B_{k}\right.\right.$ y $\lambda B_{i}$ conected $\})$. The rank max connected opening is defined, in the binary case, by:

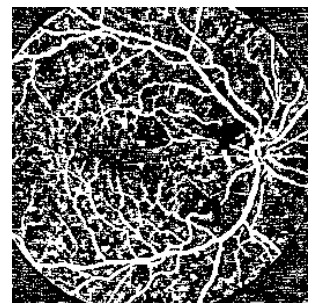

(a)

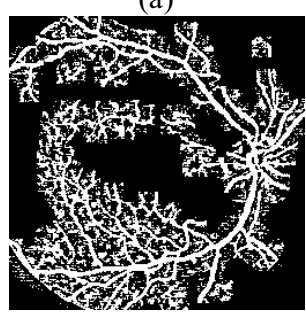

(c)

$$
\widetilde{\gamma}_{\lambda B, k}(X)=\bigcup_{i}\left\{\gamma_{\lambda B_{i}}(X): \lambda B_{i} \in B_{C k} \text { and }\left|\lambda B_{i}\right|=k\right\} \text {. }
$$

Unlike the rank-max opening, the connected rankmax opening processes independently each connected component. This means, that every connected component of an invariant of $\bar{\gamma}_{B, k}$ is itself an invariant of $\bar{\gamma}_{B, k}$. Figure 8d illustrates the output image based on a rank-max connected opening. In the gray-level case the rank-max connected opening is expressed by:

$$
\begin{aligned}
& \bar{\gamma}_{B, k}(f)(x)=\underset{i}{\vee}\left\{\gamma_{\lambda B_{i}}(f)(x): \lambda B_{i} \in B_{C k} \text { and }\left|\lambda B_{i}\right|=k\right\} \\
& \text { and } \left.\left|\lambda B_{i}\right|=k\right\}
\end{aligned}
$$

Now, it is presented the filtering process to characterize the directional structures of the fingerprints. These directional characteristics are reconstructed starting from 2 images; the first image gives the information of the predominant sizes, while the second one gives the information of the preferential orientations.

This enables to compute a set of line segments that characterize the orientation fields of the image. The application of the rank-max connected filter to the distance function enables us to decrease the number of minima in order to obtain a better description of the image by using the segments of lines as illustrated in figures $7 \mathrm{~d}$ and figure $7 \mathrm{e}$. In summary, this connected filter allows to leave alone the structures that have specific orientation and size, which are better adapted to the structures.

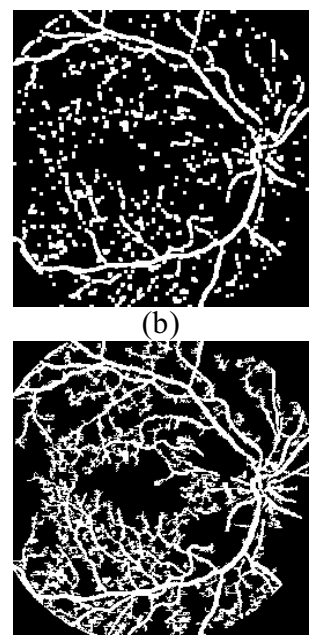

(d)

Figure 8. (a) Original Image, (b) Morphological opening size 1 ( $3 \times 3$ pixeles), (c) Rank-max opening with $\lambda=8 y k=160$, (d) Rank-max connected opening with $\lambda=8 y k=60$ 


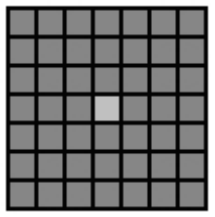

(a)

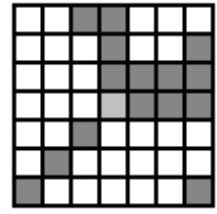

(b)

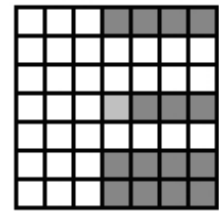

(c)

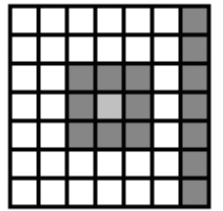

(d)

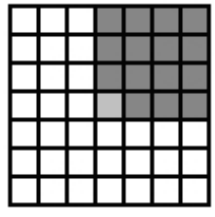

(e)

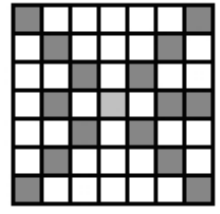

(f)

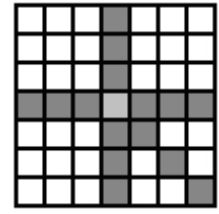

(g)

Figure 9. a) Structuring elements $\lambda B$ with $\lambda=3$ and $\left.\left.\left.\left.\left.\left|\lambda B_{i}\right|=49\left(\lambda B_{i}=\lambda B\right), b\right), c\right), d\right), e\right), f\right)$, g) Six configurations of $\lambda B_{i}$ with $\lambda=3$ and $\left.\left.\left.\left|\lambda B_{i}\right|=16, e\right), f\right), g\right)$ Structuring elements composed of only one connected component

To better illustrate the performance of this connected filtering process several tree examples figure 10a, $10 \mathrm{~d}, 10 \mathrm{f}$ and $10 \mathrm{~h}$. Here of orientation detection in fingerprints are carried out. To illustrate the orientation detection with and without filtering process, the line segment and orientation function were computed from the binary images, then a line segment was placed at each maximum of the line segment function by taking into account the direction given by the orientation function. In figure $10 \mathrm{~b}$ one illustrates the line segments representation without filtering process. Only one point of each regional maximum was selected to place a line segment. Next, a morphological filtering based on a rank-max connected opening of parameters $\mu=2, k=$ 7 was applied to the line segments function for obtaining the image in figure 10c, 10e, 10g and 10i. Observe in these last images that the filtered line segments function by a rank-max opening enables us to decrease the number of maxima, then to attenuate the information complexity of the maxima.

\section{Conclusions}

In this paper, there were presented two new methods to characterize fingerprints. First some basic concepts were presented in order to explain the proposed methods. The first one is based on the directional granulometries and the notion of quadtree structure. An innovative criterion to discriminate the directional granulometric is applied to the quadtree structure methodology. Thus, it permits to classify the orientation fields at different scales. The proposed homogeneity criterion for selecting the fields at a given hierarchy of the quadtree is specified by the principal minimum (or minima) of the distribution function of the granulometry. The second proposed method considers a local approach by using the concept of distance function. The maxima of distance function was used for computing the loci of maximal structuring elements and a second function, the orientation function, was used to obtain the angles of the line segments. This pair of local parameters enables to have a good description of the image orientation fields by means of line segments. The regional maxima of the distance function play the main role to detect the principal orientation of the structures. It was shown that with the obtained information, store as the directional fields, is possible to reconstruct the directional behavior of the fingerprint. Indeed, the maxima of the distance function enable us to determine the main structures of the fingerprint while the orientation function provide the directions of these structures. Thus, these local descriptors for the size and orientation provide an excellent reconstruction of the orientations and allow describing the model of the fingerprint orientation pattern in a piecewise manner; this means, it is possible to reconstruct de whole behavior of the ridges shape. This is carried out with only two parameters, size and orientation in some points of the main ridges.

The proposed methods were applied over a set of fingerprints with excellent results, showing robustness for segmenting directional fields. This robustness is due to the fact that our methods extract directional information from multiple scales by taking into account the whole context of the structures contained in the images. Contrary to other methods based on the gradients which are very sensitive to noise and require a final stage to enhance directional fields since they work at pixel scale. Also the main advantages observed against other 


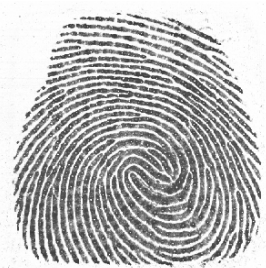

(a)

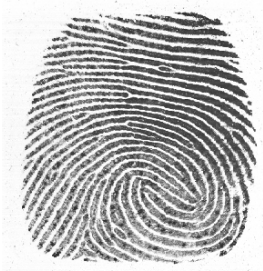

(d)

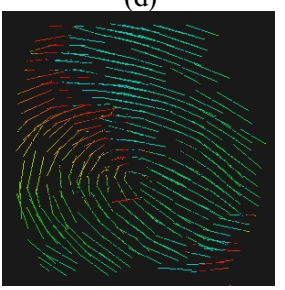

(g)

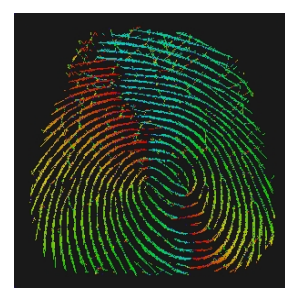

(b)

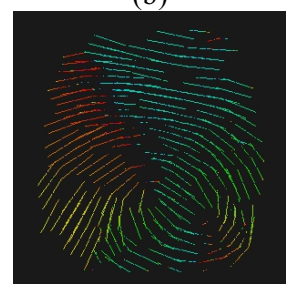

(e)

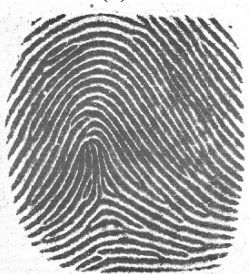

(h)

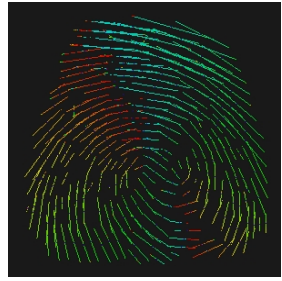

(c)

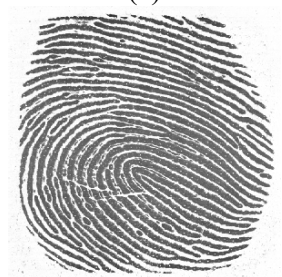

(f)

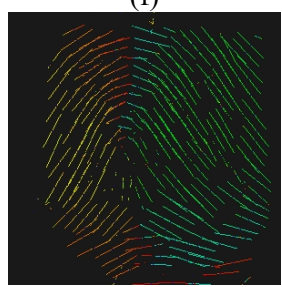

(i)

Figure 10. a) Fingerprint type whorl, b) Pre-prosecing image, c) Orientation function image, d) Line segments representation after a morphological filtering based on a rank-max (2,7), e) Fingerprint type whorl, f) Line segments representation after a morphological filtering based on a rank-max from " $e$ ", g) Fingerprint type left loop, h) Line segments representation after a morphological filtering based on a rank-max from " $g$ "

methods that seek the core of the image is that the calculus of the orientation fields are simpler and it is not necessary the detection of the core before compute them.

\section{Acknowledgments}

The first author acknowledges the government agency CONACyT for the financial support. Iván Terol would like to thank Diego Rodrigo and Darío T.G. for their great encouragement. This work was supported by de government agency CONACyT (58367) .

\section{References}

Bazen A.M., Gerez S.H. Systematic Methods for the Computation of the Directional Fields and Singular Points of Fingerprints. IEEE Trans. Pattern Anal. Mach. Intell, 24:905-919. 2002.

Cappelli R., Lumini A., Maio D., Maltoni D. Fingerprint Classification by Directional Image Partitioning. IEEE Trans. Pattern Anal. Mach. Intell, 21:402-421. 1999.
Chong M.M., Ngee T.H., Gay R.K. Geometric Framework for Fingerprint Image Classification. Pattern Recognition, 30:1475-1488. 1997.

Dass S.C. Markov Random Field Models for Directional Field and Singularity Extraction in Fingerprint Images. IEEE Trans. Image Process, 13:1358-1367. 2004.

Greenberg S., Kogan D. Improved Structure-Adaptive Anisotropic Filter. Pattern Recognition Letters, 27:59-65. 2006.

Gu J., Zhou J., Zhang D. A Combination Model for Orientation Field of Fingerprints. Pattern Recognition, 37:543-554. 2004.

Heijmans J.A.M. Morphological Image Operators. Academic Press. 1994.

Jain A.K., Hong L., Bolle R. On-line Fingerprint Verification. IEEE Trans. Pattern Anal. Machine Intell., 19:302-313. 1997a.

Jain A.K., Hong L., Pankanti S., Bolle R. An Identity-Authentication System Using Fingerprints. Proc. IEEE, 85:1365- 1431. 19976.

Jain A.K., Pankanti S., Hong L. A Multichannel Approach to Fingerprint Classification. IEEE Trans. Pattern Anal Machine Intell, 21:348-359. 1999.

Jiang X.D., Yau W.Y. Fingerprint Minutiae Matching Based on the Local and Global Structures. Proc. of the 15th International Conference on Pattern Recognition, 2:1038-1041. 2000. 
DOI: http://dx.doi.org/10.22201/fi.25940732e.2009.10n3.024

L.A. Morales-Hernández, I.R. Terol-Villalobos, A. Domínguez-González and G. Herrera-Ruiz

Karu K., Jain A.K. Fingerprint Classification. Pattern Recognition. 17:389-404. 1996.

Kawagoe M., Tojo A. Fingerprint Pattern Classification. Pattern Recognition. 17:295-303. 1984.

Khan A.U., Khan M.K., Khan M.A. Fingerprint Image Enhancement Using Decimation-free Directional Filter Bank. International Technology Journal, 4:16-20. 2005.

Kulkarni J.V., Patil B.D and Holambe R.S. Orientation Feature for Fingerprint Matching. Pattern Recognition, 39:1551-1554. 2006.

Li J., Yau W.Y., Wang H. Constrained Nonlinear Models of Fingerprint Orientations with Prediction. Pattern Recognition, 39.102-114. 2006.

Maio D., Maltoni D. Direct Gray-Scale Minutiae Detection in Fingerprints. IEEE Trans. Pattern Anal, Mach. Intell., 19: 27-40. 1997.

Maltoni D., Maio D., Jain A.K., Prabhakar S. Handbook of Fingerprint Recognition. New York. Springer Verlag. 2003.

Maragos P. Pattern Spectrum and Multiscale Shape Representation. IEEE Trans. On Pattern Analysis and Machine Intelligence, 11:701-716. 1989.

Matheron G. Random Sets and Integral Geometry. New York. Wiley. 1975.

Meyer F., Maragos P. Nonlinear Scale-Space Representation with Morphological Levelings. J. Visual Comm. Image Represent., 11:245-265. 2000.
Oliveira M.A, Leite N.J. A Multiscale Directional Operator and Morphological Tools for Reconnecting Broken Ridges in Fingerprint Images. Pattern Recognition, 41:367-377. 2007.

Qi J., Wang Y. A Robust Fingerprint Matching Method. Pattern Recognition, 38:1665-1671. 2005.

Ratha N., Chen S., Jain A.K. Adaptive Flow Orientation Based Feature Extraction in Fingerprint Images. Pattern Recognition, 28:1657-1672. 1995.

Serra J. Image Analysis and Mathematical Morphology. J. Serra, Ed. Vol. II. New York. Academic Press. 1988.

Sherlock B., Monro D. A Model for Interpreting Fingerprint Topology. Pattern Recognition, 26:1047-1055. 1993.

Soille P. Morphological Image Analysis. Heidelberg. SpringerVerlag. 2003.

Soille P., Talbot H. Directional Morphological Filtering. IEEE Trans. on Pattern Anal. Machine Intel, 33:1313-1329. 2001.

Vizcaya P.R., Gerhardt L.A. A Nonlinear Orientation Model for Global Description of Fingerprints. Pattern Recognition, 29:1221-1231. 1996.

Zhou J., Gu J. A Model-Based Method for the Computation of Fingerprints Orientation Field. IEEE Trans. Image Process, 13: 821-835. 2004.

Zhou J., Gu J. Orientation Fields of Fingerprints with Rational Complex Functions. Pattern Recognition, 37:389391. 2004.

\footnotetext{
About the authors

Luis A. Morales-Hernández. Is a Ph.D. student and professor in the engineering faculty in the Autonomous University of Queretaro (UAQ). He received his B.Sc. in electromechanics engineering and M.Sc. degree in Instrumentation and Control Automatic with commendation from the University Autonomous of Queretaro. His current research interests are image segmentation, granulometries morphological and measured microstructure in metals.

Iván R. Terol-Villalobos. He received his BSc degree from Instituto Politécnico Nacional (I.P.N. México), his MSc degree in Electrical Engineering from Centro de Investigación y Estudios Avanzados del I.P.N. (México), and a DEA in Computer Science from the University of Paris VI (France). He received his PhD degree from the Centre de Morphologie Mathématique, Ecole des Mines de Paris (France). Currently, he is a researcher at Centro de Investigación y Desarrollo Tecnológico en Electroquímica (Querétaro, México). His main current research interests include morphological image processing, morphological probabilistic models and computer vision.

Aurelio Dominguez-González. He is an electromechanical engineer graduated from the University Autonomous of Queretaro (UAO) in Mexico. He studied the M. Sc. in mechanical engineering in the Instituto Tecnologico de Queretaro, Mexico (ITQ) and he received the $\mathrm{Ph}$. D. in mechanical engineering from the Concordia University in Montreal, Canada. Actually, he is professor and researcher of the Engineering Faculty of UAO. His main current research interests are the structural optimization, automatization of manufacture machines and image processing.

Gilberto Herrera-Ruiz. He received his BSc degree in Electronic System Engineering, his MSc degree in Electrical Engineering and his $\mathrm{PhD}$ degree Mechanical Engineering. he is member of the National System of Investigators in the Level II. His is current director of the Faculty of Engineering in the Autonomous University of Queretaro. His main current research interests are automatization of manufacture machines and image processing.
} 